\title{
On Mobile Agent Positioning for Wireless Network Reconfiguration
}

\author{
Athanasia Panousopoulou \\ Department of Electrical and Computer Engineering \\ University of Patras \\ Rio, GR-26500, Greece \\ Email: apanous@ece.upatras.gr
}

\author{
Anthony Tzes \\ Department of Electrical and Computer Engineering \\ University of Patras \\ Rio, GR-26500, Greece \\ Email: tzes@ece.upatras.gr
}

\begin{abstract}
Partially connected heterogeneous wireless sensor networks (hWSNs) can be reconfigured via the utilization of mobile agents that aim in reconnecting the disconnected clusters of nodes. Under the assumption of the knowledge of the nodes' spatial location and the transmitting power of their uniform radiation pattern, the presented algorithm aims at optimizing the positioning of a limited number of mobile agent for the establishment of the connectivity among the disjoint clusters of nodes. The developed algorithms maximize their corresponding objective functions, whose primary terms are related to (a) the cardinality of each cluster of nodes and (b) the minimum distance between the clusters. The extended simulation results are offered to evaluate the performance of the proposed solutions.
\end{abstract}

\section{INTRODUCTION}

Wireless Sensor Networks (WSN) [1-3] combine sensing, data processing and communicating in a unified platform. Their distributed operational philosophy results in networking environments, where the participating nodes are self-aware, self-healing and self-organized; their autonomy enhances our ability to monitor the physical environment, even in topologies/situations where the human access is not always feasible [4-6].

One of the major issues related to the WSN is the nodes' deployment; the determination of the optimal geographical topology that would ensure coverage and network connectivity [7-9] is of paramount importance. Major contributions can be found in the recent literature for the coverage problem in static and mobile sensor networks. The efficiency of coverage algorithms for static networks based on persistent homology theory is illustrated in [10]. In most cases, basic computational geometry concepts [11] are utilized for solving coverage

This work was partially funded by the European Social Fund (75\%), the Greek Secretariat's Research and Technology (25\%) and ATMEL HELLAS S.A., within the framework of Measure 8.3 of the Operational Programme "Competitiveness" and the 3rd Community Support Programme (PENED03868). problems in static and mobile WSN. Typical examples of efficient coverage algorithms based on Delaunay Triangulation and Voronoi Diagrams can be traced at [1215]. Another alternative related to the dynamic coverage for mobile sensor networks based on graph theory and High order Laplacians can be found at $[16,17]$, while distributed motion coordination algorithms for multiple vehicle and sensor networks are surveyed at [18].

The effort presented in this paper is the sequel of the reconfiguration concept for a static heterogeneous WSN (hWSN), based on Voronoi diagrams. The reconfiguration process of the proposed scheme serves two objectives: (a) guarantee the radio coverage within the bounds of the geographical area, and (b) ensure the connectivity among the operating nodes. To assist in the fulfilment of this objective a number of mobile agents (robots) is introduced into the underlying Voronoi topology in order to establish connectivity among the isolated clusters of nodes.

This work focuses on the mobile agent placement process, while several other enhancements related to the qualification of the status of the Voronoi-based network, the modeling of the nodal radio coverage, the radio connectivity definition, and the nodes' spatial deployment are also offered. The mobile agent placement process presented at an earlier work ([19]) is extended and, as a result, the combined algorithm is formed.

This paper is organized as follows: The mathematical framework of the Voronoi-modeled network is defined in Section II. The revisited network reconfiguration algorithm is outlined in Section III, followed by the simulation results (Section IV). Concluding remarks are offered in the last Section.

\section{Problem Statement}

In the present work, we consider a sensor network residing within a bounded geographical area, already operating for some time period. The communication objective, of this sensor network is twofold; first of all, it should provide radio "hospitality" to every device that temporally resides within the network's geographical 
area. Moreover, it should yield a qualitative connectivity status.

The geographical area's coverage is a task distributed among the operational nodes, and results in the area fragmentation into regions. Each node is assigned with one region and becomes responsible for the radio coverage within the latter's bounds. This task allocation is not in the general case uniformly distributed and combined with the nodal heterogeneity, may result in a highly unfair networking environment.

The geographical mapping for the aforementioned responsibility regions' allocation is modeled as a Voronoi $V_{N}$ diagram, whose $N$ sites are the operational nodes. The basic assumptions for this Voronoi-based network, provide an adequate knowledge of the network's status. As already mentioned a static network is considered, with known coordinates for the sites. The signal propagation over the wireless medium is modeled by a deterministic radio propagation model ([20]). Consequently, a cyclic radiation pattern is assumed for each node. From a "networked" Voronoi-topological point of view, each node, $i \in N$, is characterized by:

1) The static coordinates $\bar{c}_{i}=\left(x_{i}, y_{i}\right)$.

2) The transmission coverage area $C_{i}=$ $\left\{(x, y): \sqrt{\left(x-x_{i}\right)^{2}+\left(y-y_{i}\right)^{2}} \leq r_{i}\right\}$, where $r_{i}$
is the transmission range of node $i$.

3) The Delaunay neighbors set [11] defined by $\Delta_{i} \subset\{1, \ldots, i-1, i+1, \ldots, N\}$.

4) The vertices set defining its border of responsibility $\tilde{P}_{i}=\left\{\bar{u}_{i 1}, \bar{u}_{i 2}, \ldots, \bar{u}_{i k}\right\}$.

For each node, let the intersection of its responsibility area within its coverage area be denoted as:

$$
I_{i}=P_{i} \cap C_{i},
$$

where $P_{i}$ is the convex hull representation of the polygon defined be the vertex-set $\tilde{P}_{i}$. Node $i$ is termed to be connected with node $j \in \Delta_{i}$ if messages can be exchanges between them, or expressed in range radius terms, if:

$$
d_{i j}=\left\|\bar{c}_{i}-\bar{c}_{j}\right\| \leq \min \left\{r_{i}, r_{j}\right\} .
$$

Let the members of the set $\Omega_{i}^{C}\left(\Omega_{i}^{U}\right)$ be the Delaunay neighbors sets to which node $i$ can (cannot) communicate, or

$$
\begin{aligned}
& \Omega_{i}^{C}=i,\left\{j \in \Delta_{i}: d_{i j} \leq \min \left\{r_{i}, r_{j}\right\}\right\} \\
& \Omega_{i}^{U}=\Delta_{i}-\Omega_{i}^{C} .
\end{aligned}
$$

Moreover, a cluster of nodes is called "connected" if any node within its membership list can exchange messages with another node in this cluster. Finally, the number of disjoint clusters is noted as $M$, where $1 \leq M \leq N$, and the cardinality $L_{i}$ of each cluster is defined as the number of nodes that belong to it. A complete example of a Voronoi-modeled network is presented in Figure 1.

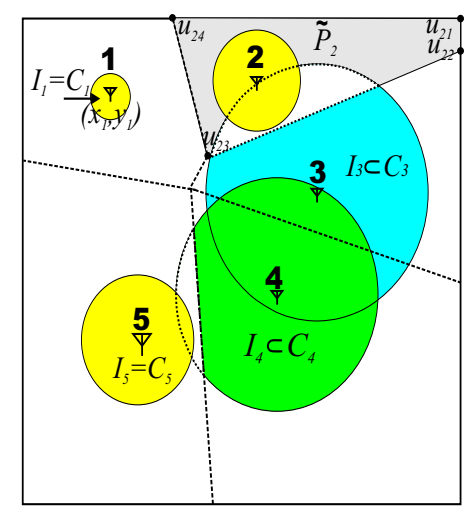

Fig. 1. An example of the Voronoi-modeled network. The transmission areas are denoted by the circles centered around the nodes' coordinates $\bar{c}_{i}, i=1, \ldots, 5$, while for the second node the Delaunay neighbors set is $\Delta_{2}=\{1,3\}$ and its responsibility region is $\tilde{P}_{2}=\left\{u_{2 j}\right.$, for $\left.j=1, \ldots, 4\right\}$, depicted by the grey polygon. The active areas are $I_{i}=C_{i}, i=1,2,5$, while $I_{3} \subset C_{3}$ and $I_{4} \subset C_{4}$. Finally, there are four clusters with membership $\Gamma_{1}=\{1\}, \Gamma_{2}=\{2\}, \Gamma_{3}=$ $\{3,4\}, \Gamma_{4}=\{5\}$. The number of disjoint clusters is $M=4$, while the cardinality $L_{i}$ for each cluster $i$ is $L_{i}=1$ for $i=1,2,4$ and $L_{i}=2$ for $i=3$.

In the present work, the primary objective of the network reconfiguration policy is the minimization of the number of isolated network clusters. Towards this goal, the approach adopted aims at establishing the connectivity among disjoint clusters, by introducing a limited number of mobile agents that are not a part of the Voronoi topology. As a side effect of the mobile agent's placement, the proposed scheme also calculates the reduction of the area of the "blind" regions, i.e. the regions that are not covered from the transmission range area of the operational sites. In the sequel the mathematical framework for the mobile agent positioning and the blind region calculation is provided.

\section{A. Mobile Agent Positioning}

The mobile agent placement problem has to be solved into two discrete steps. The first step involves determining the optimal position for placing a mobile agent in order to join two isolated clusters. The second step involves the selection of the optimal cluster pair to be joined given the limited resources on the number of mobile agents. In this section, the first step is examined; the selection of the optimal cluster pair will be analyzed in Section III.

1) Calculation of two clusters' distance: Let $\Gamma_{\mu}$ and $E_{\mu}$ the sites-set and the connected edges set of the $\mu$-th cluster respectively. A connected edge $e_{i j}$ is defined as the sites pair $(i, j)$ that are connected to each other according to the connectivity definition given by (2). More specifically, the $E_{\mu}$ set is determined by the following equation:

$$
E_{\mu}=\left\{e_{i j}=(i, j), i, j \in \Gamma_{\mu}, i \neq j: d_{i j} \leq \min \left\{r_{i}, r_{j}\right\}\right\}
$$


The distance $d_{i e_{j k}}$ among a site $i \in \Gamma_{\lambda}$ and a connected edge $e_{j k} \in E_{\mu}$, is based on the calculation of the height $h$ of the triangle defined by the points $\left\{\bar{c}_{i}, \bar{c}_{j}, \bar{c}_{k}\right\}$ and originated by the $i$-th site.

$$
d_{i e_{j k}}=\left\{\begin{array}{l}
|h|, \text { if } \bar{c}_{h} \in\left[\bar{c}_{j}, \bar{c}_{k}\right] \\
\min \left\{d_{i j}, d_{i k}\right\}, \text { otherwise }
\end{array},\right.
$$

where the $|$.$| operand defining the magnitude of the \vec{h}$ vector, and $\bar{c}_{h}$ are the coordinates of the trace of the height $h$. The coordinates $\bar{c}_{e_{1}}, \bar{c}_{e_{2}}$ of the extremes $\left(e_{1}, e_{2}\right)$ of the line segment defined by the distance $d_{i e_{j k}}$ are determined according to equations set:

$$
\begin{aligned}
& \bar{c}_{e_{1}}=\bar{c}_{i} \\
& \bar{c}_{e_{2}}= \begin{cases}\bar{c}_{h}, & \text { if } \bar{c}_{h} \in\left[\bar{c}_{j}, \bar{c}_{k}\right] \\
\bar{c}_{h^{\prime}} & \text { otherwise. }\end{cases}
\end{aligned}
$$

The point $h^{\prime}$ stated at (8) is defined as: $h^{\prime} \in$ $\{j, k\}$ and $d_{i h^{\prime}}=\min \left\{d_{i j}, d_{i k}\right\}$. The distance $d_{\lambda \mu}$ among the $\lambda$-th and $\mu$-th clusters is determined by the minimum distance among the sites $\in \Gamma_{\lambda}$ and the connected edges $\in E_{\mu}$, based on (6):

$$
d_{\lambda \mu}=\min _{\substack{i \in \Gamma_{\lambda} \\ e_{j k} \in E_{\mu}}} d_{j k} .
$$

Given that the mobile agents' transmission power is fixed, the number of the mobile agents required to establish connectivity of the $\lambda-\mu$ cluster pair (distance weight factor) is defined as:

$$
w_{\lambda \mu}=\left\lceil\frac{d_{\lambda \mu}-\min \left\{r_{i}, R_{a}\right\}}{R_{a}}\right\rceil,
$$

where $i$ is the site $\in \Gamma_{\lambda}$ for which the distance among the $\lambda$-th and $\mu$-th clusters is minimum, $r_{i}, R_{a}$ are the transmission radii of node $i$ to the mobile agent respectively.

2) Mobile agent's placement: The mobile agent's desired position should ensure its connectivity with at least one of the clusters involved. Moreover, it should reside within the line segment defined by the clusterpair's distance, given at (9). Consequently, the mobile agent's coordinates $\left(\bar{c}_{a}=\left(x_{a}, y_{a}\right)\right)$ should satisfy the following set of (in)equalities:

$$
\begin{array}{r}
\left\|\bar{c}_{e_{1}}-\bar{c}_{a}\right\|_{2} \leq \min \left\{r_{e_{1}}, R_{a}\right\} \\
y_{a}=\frac{y_{e_{1}}-y_{e_{2}}}{x_{e_{1}}-x_{e_{2}}} x_{a}+y_{e_{1}}-\frac{y_{e_{1}}-y_{e_{2}}}{x_{e_{1}}-x_{e_{2}}} x_{e_{1}}
\end{array}
$$

where $\bar{c}_{e_{1}}=\left(x_{e_{1}}, y_{e_{1}}\right)$ and $\bar{c}_{e_{2}}=\left(x_{e_{2}}, y_{e_{2}}\right)$ are the coordinates of extremes (7-8) of the line segment defined by the cluster's distance. The selection of the representative site for each cluster is based on the cluster-pair distance, described by (9). The equation set (11)-(12), will provide two solutions for the agent's coordinates. The accepted solution is the one lying among the $\mu-\lambda$ cluster pair.

\section{B. Blind Region Calculation}

The blind region $\left(P_{i}^{b}\right)$ of a polygon $P_{i}$ determines the subarea of $P_{i}$ that cannot provide radio "hospitality" to any device that might temporally reside within it. In radio coverage terms, the blind region is the subarea of the $P_{i}$ that is 0-times "radio covered", whereas the active region is the subarea of the $P_{i}$ that is $m$-times radio-covered, where $1 \leq m \leq N$. In the present work, the metric utilized for quantifying the blind region of a Voronoi polygon $P_{i}$ is based on calculating the latter's surface that remains uncovered from the transmission range of the operational nodes.

More specifically, let $I_{i j}$ be the intersection of $C_{j}$ with $P_{i}$, where $j \in N$ :

$$
I_{i j}=P_{i} \bigcap C_{j} .
$$

The active region $I_{i}^{N}$ of the $P_{i}$ extends the definition given by (1) and is determined as the union of all the transmission range areas intersected with the $P_{i}(13)$ :

$$
I_{i}^{N}=\bigcup_{j \in N} I_{i j}=P_{i} \bigcap\left(C_{1} \bigcup C_{2} \bigcup \ldots \bigcup C_{N}\right) .
$$

The quantification of the blind region $P_{i}^{b}$ is based on calculating the area covered by $P_{i}^{b}$; for this reason, let the operator $A\left(P_{i}^{b}\right)$ be defined as the area occupied by the spatial set $P_{i}^{b}$. More specifically, the area $A\left(P_{i}^{b}\right)$ is the difference between the area of polygon $P_{i}$ and the area of the latter's active region $\left(A\left(I_{i}^{N}\right)\right)$, or:

$$
A\left(P_{i}^{b}\right)=A\left(P_{i}\right)-A\left(I_{i}^{N}\right) .
$$

The proper calculation of $A\left(I_{i}^{N}\right)$ is based on carefull consideration regarding the overlapping areas $I_{i j}, j=$ $1,2 \ldots N$ within the $P_{i}$ region. Towards this direction, $A\left(I_{i}^{N}\right)$ equals to the sum of the areas of $I_{i j}$ regions covered exclusively by only one subregion of $I_{i j}\left(\bar{A}\left(I_{i j}\right)\right)$, as described in (15):

$$
A\left(I_{i}^{N}\right)=\sum_{j=1}^{N} \bar{A}\left(I_{i j}\right) .
$$

The calculation of $\bar{A}\left(I_{i j}\right)$ requires the determination of the successively intersected areas among the $I_{i j}(j=$ $1 \ldots, N)$ regions for the $P_{i}$, as shown in the sequel:

$$
\begin{aligned}
& \bar{A}\left(I_{i j}\right)=A\left(I_{i j}\right)-\sum_{k=j+1}^{N} A\left(I_{i j} \cap I_{i k}\right)+ \\
& \sum_{k=j+1}^{N-1} \sum_{l=k+1}^{N} A\left(I_{i j} \cap I_{i k} \cap I_{i l}\right)- \\
& \sum_{k=j+1}^{N-2} \sum_{l=k+1}^{N-1} \sum_{n=l+1}^{N} A\left(I_{i j} \cap I_{i k} \cap I_{i l} \cap I_{i n}\right)+\ldots+ \\
& (-1)^{m} \sum_{k=j+1}^{N-m} \ldots \sum_{n=l+1}^{N-1} \sum_{v=n+1}^{N} A\left(I_{i j} \cap I_{i k} \ldots \bigcap I_{i n} \cap I_{i v}\right)
\end{aligned}
$$

The $m$-factor $(m \in \mathbb{Z})$ indicates the level of radio coverage of the $I_{i j}$ subregion; for $m=n$, a region is covered by subregions of $n$ sites' transmission ranges. 


\section{REVISED NETWORK RECONFIGURATION ALGORITHM}

The Network Reconfiguration policy presented and evaluated in our previous work has a twofold objective; (a) the minimization of the blind region areas and (b) the minimization of the disjoint clusters. The effort made here aims at improving the original concept ([19]), with the focus made on the connectivity issues.

The underlying assumption of the Network Reconfiguration (NetRec) algorithm is the deployment of $N$ nodes within the bounds of a geographical rectangular surface $\mathbf{G}$ with area $\mathbf{G}_{\mathbf{w}} \times \mathbf{G}_{\mathbf{w}}$. Each node has cyclic transmitting and receiving (sensing) pattern, whose radius is $r_{s}$. Ideally, the number of nodes, $N$, required to cover the area $\mathbf{G}$ without any overlapping in their sensing areas is $N=\left[\frac{\mathbf{G}_{w} \mathbf{G}_{w}}{\pi r_{s}^{2}}\right]$. In the ensuing simulation studies, $N$ nodes are randomly deployed within the area $\mathbf{G}$ at the locations $\overline{c_{1}}, \overline{c_{2}}, \ldots, \overline{c_{N}}$. Let $\overline{\mathbf{c}}=\left[\overline{c_{1}}, \overline{c_{2}}, \ldots, \overline{c_{N}}\right]$, be the array of coordinates of the deployed sensors, which is known a priori.

The core of the revisited NetRec algorithm is completed into 3 steps: (a) Voronoi Modeled Network Mapping, (b)Active Area and Cluster Set Calculation and (c) Mobile Agents Placement Algorithm.

During the first step of the algorithm the mapping of the Voronoi-modeled network is derived, based on the sites' coordinates, $\left(\overline{\mathbf{c}}=\left[\bar{c}_{1}, \bar{c}_{2}, \ldots, \bar{c}_{N}\right]\right)$ and the bounds of the geographical area $\mathbf{G}$. The results of this step step are (a) the vector of the Voronoi polygons bounded by $\mathbf{G} \mathbf{P}=\left[\begin{array}{llll}P_{1} & P_{2} & \ldots & P_{N}\end{array}\right]$, and (b) the Delaunay neighbors superset $\Delta=\left[\Delta_{1}, \Delta_{2}, \ldots \Delta_{N}\right]$ for the $V_{N}$ diagram.

The second step of the NetRec algorithm aims at the calculation of the active areas set $\mathbf{I}$ and the clusters set $\mathbf{M}$ for the Voronoi $V_{N}$. The parameters involved are the set of polygons $\mathbf{P}$, the Delaunay superset $\Delta$ (defined at the previous step), and the transmission range set $\mathbf{C}$ for all the $V_{N}$ 's cites: $\mathbf{C}=\left[C_{1}, C_{2}, \ldots C_{N}\right]$. The $\mathbf{I}$ set is calculated by (13) for each $i \in N: \mathbf{I}=\left[I_{1}, I_{2} \ldots, I_{N}\right]$.

The set of clusters $\mathbf{M}$ contains the isolated clusters, derived by (3)-(4) for each site $i$, based on the $\Delta$ superset. Moreover, during this step, the blind region of the Voronoi diagram is determined, by gathering all the surfaces $A\left(P_{i}^{b}\right)$ (14) into the vector $\mathbf{A}\left(\mathbf{P}^{b}\right)=\left[A\left(P_{1}^{b}\right) A\left(P_{2}^{b}\right) \ldots A\left(P_{N}^{b}\right)\right]$.

The clusters set $\mathbf{M}$ accompanied by the number of the available mobile agents, are the input parameters for the final step of the NetRec algorithm. The decision related to the optimal cluster pair to be joined given the constraints of the available mobile agents is derived based on the combination of two criteria and will be described in the Section III-A. The calculation of a mobile agent's position $\bar{c}_{a}$ is derived by (11)-(12), while when the latter is placed into the Voronoi diagram, the clusters set $\mathbf{M}$ is recalculated. This process is repeated for the next mobile agent. The final step terminates when (a) the number of clusters $M$ has reduced into 1 , or (b) the number of agents available $R$ has reduced into 0 .

\section{A. Selection of the optimal cluster-pair}

The selection of the optimal cluster-pair is made based on the combination of two criteria; (a) the number of mobile agents required to join the cluster-pair, already described at Section II-A1 and (b) each clusters cardinality.

The cardinality $L_{\mu}$ of a cluster $\mu$ is defined as the cluster's size in terms of containing sites. For the $\mu-\lambda$ cluster pair, with $\mu \neq \lambda$, the cardinality is given by:

$$
L_{\mu \lambda}=L_{\mu}+L_{\lambda} .
$$

As already stated, two algorithms for the selection of the optimal cluster pair (plain and combined) will be evaluated. In the following subsections the features of each algorithm are presented.

1) Plain Algorithm: The combination of the distance weight factor (10) and cardinality metrics (16) for determining the optimal cluster pair results frequently into a compromise among the aforementioned criteria; the smaller the $w_{\mu \lambda}$ the less mobile agents "consumed", while the higher the $L_{\mu \lambda}$ the bigger the number of sites connected. The level of the trade-off is strongly attached to the spatial distribution and the transmission range of the operational nodes. The solution proposed in our previous work is summarized at maximizing the following cost function:

$$
\beta_{L} \max L_{\mu \lambda}-\beta_{w} \min w_{\mu \lambda},
$$

where the weights $\beta_{L}, \beta_{w}, \in \mathbb{R}^{+}$.

The cost function described by (17) is modified to formulate the combined algorithm for the mobile agents placement process, outlined in the sequel.

2) Combined Algorithm: The combined algorithm is divided into two basic steps: (a) the selection of an original "optimal" cluster pair, and (b) the exhaustive search around the coefficients the aforementioned cluster pair for further improvement on the network's status.

During the first step, the distance and cardinality criteria are combined under the constraint of the number of mobile agents available for for the reconfiguration process. The objective of the network is summarized at maximizing the modified cost function described by (18), under the limitation of the available number of mobile agents. This constraint is described by (a) their initial number $R$, (b) the amount of those already consumed $\alpha$ and (c) the minimum distance weight factor $w_{\min }$ for all cluster pairs. The cost function, $\forall i, j \in M, i \neq j$, is:

$$
\left\{\begin{array}{c}
\beta_{L} L_{i j}+\beta_{w}\left(R-\alpha-w_{i j}\right), \text { if } R-\alpha-w_{\min } \geq 0 \\
\beta_{L} L_{i j}-\beta_{w} w_{i j}, \text { with } \beta_{L} \gg \beta_{w} \text { elsewhere }
\end{array}\right.
$$

Note that if the constraint cannot be satisfied, i.e if the minimum distance weight factor $w_{\min }$ is higher than the actual number of available mobile agents, then the policy 
of the plain algorithm described by (17) is adopted. A typical pseudocode for the first step of the combined algorithm is outlined at Table I.

TABLE I

COMbined Algorithm: Selection of ORIGinal Optimal Cluster Pair

WHILE $M>1$ AND $\alpha<R$ :

\section{$\forall\{i, j\} \in M$ :}

1. Calculate $w_{i j}$ and $L_{i j}$ from (10) and (16) respectively.

2. Calculate the minimum distance weight factor

$\min _{\{i, j\} \in M} w_{i j}=w_{\min }$ and go to step 3

3. IF $w_{\min } \leq R-\alpha$ THEN

3a. Find the cluster pair $\{\mu, \lambda\}$ that maximizes the upper right term of (18).

ELSE

3b. Find the cluster pair $\{\mu, \lambda\}$ that maximizes the

lower right term of (18).

END

Proceed to step 4 with the cluster pair $\{\mu, \lambda\}$ selected.

4. Calculate the mobile agent's coordinates $\left(x_{a}, y_{a}\right)$ according to

(11)-(12) and go to step 5.

5. Place the mobile agent into the network topology and go to step 6 .

6 . Update the number of "consumed" mobile agents according to:

$\alpha=\alpha+1$ and go to step 7 .

7. Recalculate the number $M$ of connected clusters connected clusters, with the mobile agent considered as an operational node.

END

During the second step of the combined algorithm, an exhaustive search around the "neighborhood" of the optimal cluster pair's coefficients is performed. More specifically, given the "optimal" cluster pair $c=\{\mu, \lambda\}$ (with the procedure outlined at Table I) that maximizes (18), the combination of clusters around the "neighborhood" of the clusters $\mu$ and $\lambda$ providing better results in terms of cardinality and mobile agents consumed is to be examined. The neighborhood $c_{i}^{M}$ of the cluster $i \in c$ is defined as the set of clusters $j \in M / c$ such that:

$$
w_{i j} \leq w_{\mu \lambda} .
$$

More specifically, the $c_{i}^{M}$ set is determined by the following equation:

$$
c_{i}^{M}=\left\{j \in M / c: w_{i j} \leq w_{\mu \lambda}\right\}
$$

If $c_{i}^{M}$ is a non-empty set, then the combined algorithm examines whether the union of the $i$-th cluster with several of its neighbors $j$ can generate an augmented cluster $(\tilde{i})$ that is more "popular" in terms of cardinality $\left(L_{\tilde{i}}\right)$ with less mobile agents $\left(w_{\tilde{i}}\right)$ required, than the optimal cluster pair $c$. This process, repeated for both clusters $\mu, \lambda \in c$, results into two augmented clusters $\tilde{\mu}$ and $\tilde{\lambda}$. The cost function formed (20), is based on the initial network reconfiguration process (17):

$$
\beta_{L} L_{\tilde{i}}-\beta_{w} w_{\tilde{i}}, \tilde{i} \in\{\tilde{\mu}, \tilde{\lambda}\} .
$$

A typical pseudocode for the second step of the combined algorithm is outlined at Table II.

\section{Simulation Studies}

The NetRec algorithm explained at the previous Section has been tested for several cases of square geographical areas. For testing a wide range of networks in each case the square area's width is set in a recursive manner as: $\mathbf{G}_{w}(i)=\mathbf{G}_{w}(i-1)+\Delta \mathbf{G}_{w}(i-1)$ and $\Delta \mathbf{G}_{w}(i)=\Delta \mathbf{G}_{w}(i-1)+1$. The index $i=1, \ldots, 9$ (nine runs) corresponds to the index of the examined area, while for $i=0 \mathbf{G}_{w}(0)=12$ and $\Delta \mathbf{G}_{w}(0)=5$. For a more representative simulation study the number of scattered nodes $N(i)$, under the assumption of a sensing radius of $r_{s}=5$. Furthermore for normalization purposes, the number of available mobile agents is defined as

$$
R(i)=\frac{2}{M(i)(M(i)-1)} \sum_{\substack{\lambda=1 \\ \mu=\lambda+1 \\ \lambda \neq \mu}}^{\frac{M(i)(M(i)-1)}{2}} w_{\lambda \mu}(i),
$$

where these robots are used to join the $\frac{M(i)(M(i)-1)}{2}$ cluster-pairs for each case, and $w_{\lambda \mu}(i)$ is the distance weight factor of the ' $\lambda \mu$ ' pair for the $i$ th case. Based on these "normalized" cases, the width, number of stationary nodes and mobile agents for all cases, along with the remaining parameters are provided in Table III.

TABLE III

SimUlation PARAMETERS

\begin{tabular}{|l|l|}
\hline Simulation Parameters & Value \\
\hline Geographical Area $\left(\mathbf{G}_{w}(i) \mathbf{G}_{h}(i)\right)$ & $289,529,900,1444,2209$, \\
& $3244,4624,6400,8649$ \\
\hline Network Size $(N(i))$ & $4,7,12,19,29$, \\
& $42,59,82,111$ \\
\hline Number of spatial topologies & \\
examined for each network case $i$ & 50 \\
\hline Sensing Radius $\left(r_{s}\right)$ & 5 \\
\hline Radio Signal Propagation Model & Deterministic \\
\hline Operational Frequency & $2.4 \mathrm{GHz}$ \\
\hline Receiver's antenna sensitivity $(S)$ & $-94 \mathrm{dBm}$ \\
\hline Transmission Power $\left(\forall i \in V_{N}\right)$ & $-25 \mathrm{dBm}$ \\
\hline Mobile Agents Available $\left(R_{i}\right)$ & $4,4,6,7,8$, \\
& $10,11,13,15$ \\
\hline Antennas gain $\left(G_{t x}, G_{r x}\right)$ & 1 \\
\hline Losses in antenna $(L)$ & 1 (No losses) \\
\hline Antennas height $\left(h_{t x}, h_{r x}\right)$ & $0.015 \mathrm{~m}$ \\
\hline Mobile Agent Transmission Power & $-25 \mathrm{dBm}$ \\
\hline
\end{tabular}

\section{A. Simulation Results}

The efficiency of the NetRec algorithm is examined for both the plain and combined algorithms for the mobile agent reconfiguration process described at the previous Section. The combination of the cardinality and distance criteria via the cost functions (18) and (20) is implemented considering that the priority is on the cardinality criterion, i.e. $\beta_{L} \gg \beta_{w}$.

The metrics utilized for evaluating the performance of each algorithm are (a) the number of isolated clusters, 
TABLE II

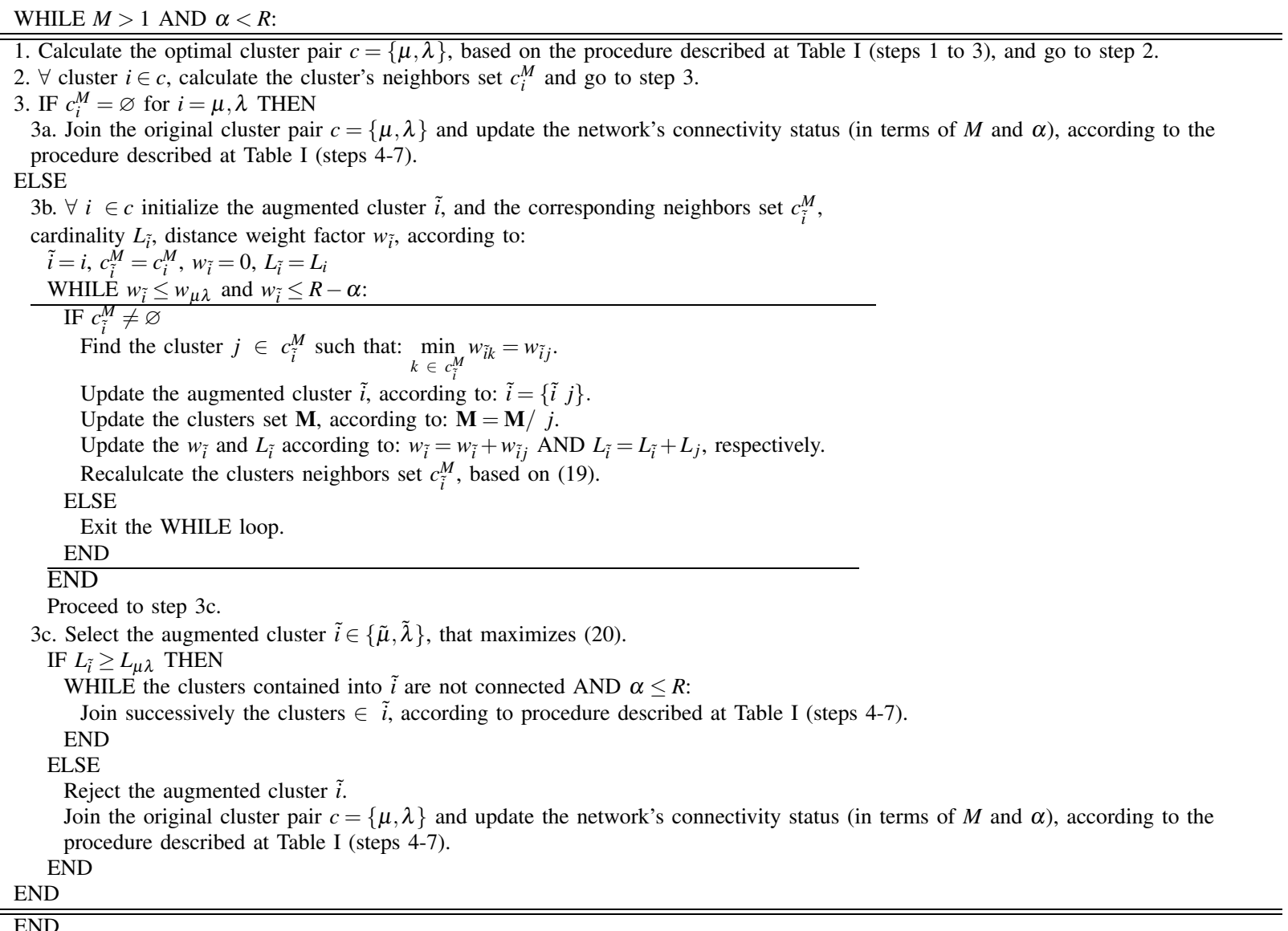

(b) the total number of mobile agents required to reduce $M$ to 1 and (c) the $V_{N}$ 's blind region surface, after the algorithm's termination.

The normalized number of isolated clusters $\gamma_{M}$ is defined as $\gamma_{M}=\frac{M_{\text {final }}}{M_{\text {initial }}}$, where $M_{\text {initial }}$ and $M_{\text {final }}$ are the number of isolated clusters before and after the mobile agent's placement process respectively. The $\gamma_{M}$ metric for the network cases examined is depicted at Figure 2. The mean value of $\gamma_{M}$ accompanied by the minimum and maximum values for each network case highlights the fact that the combined algorithm provides better results in terms of connectivity. Especially for $N \geq$ 7 , the mean value of $\gamma_{M}$ remains constantly higher for the combined algorithm case. Moreover, the maximum value for $\gamma_{M}\left(\max \gamma_{M}=1\right)$, i.e. the worst connectivity status after the termination of NetRec, is observed for the plain algorithm case, even for small and medium sized networks $(7 \leq N \leq 29)$. Although the results indicate that adopting the combined algorithm results into more clusters connected to each other, none of the algorithms presented here cannot establish full connectivity among all the operational nodes. This phenomenon is strongly attached to the limitation on the number of mobile agents

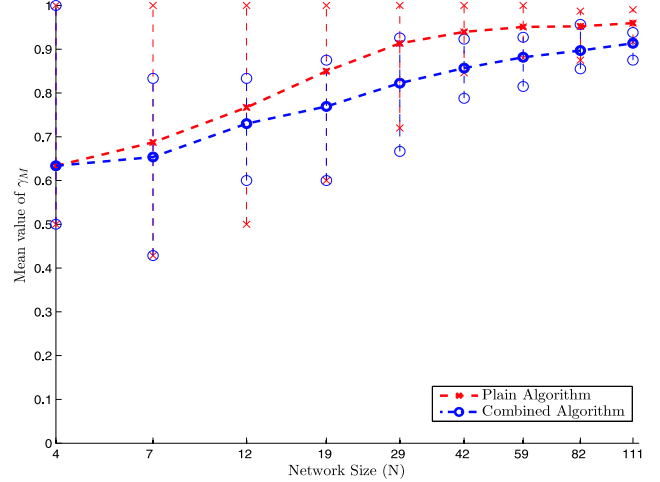

Fig. 2. The mean value of the normalized number of isolated clusters for (a) the plain and (b) the combined algorithm, after the termination of the network reconfiguration policy versus the network's size.

available for full network connectivity restore.

Prior to the initialization of NetRec, the maximum number of mobile agents needed to restore connectivity 
between every possible pair of disconnected clusters is

$$
w_{\text {initial }}=\sum_{\substack{\lambda=1 \\ \mu=\lambda+1 \\ \lambda \neq \mu}}^{\frac{M(i)(M(i)-1)}{2}} w_{\lambda \mu}(i) .
$$

Similarly, after the application of NetRec, this number $w_{\text {final }}$ is reduced. Henceforth, the relative number of mobile agents required to completely restore the network connectivity after versus prior the application of NetRec is defined as $\gamma_{W}=\frac{w_{\text {final }}}{w_{\text {initial }}}$. The results are highlighted at Figure 3. The combined algorithm outperforms in

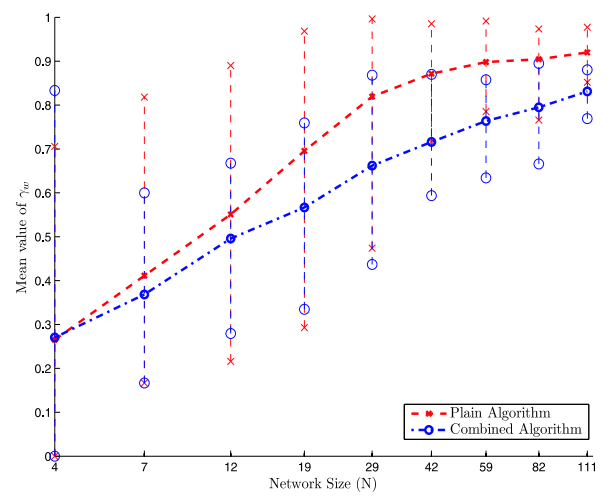

Fig. 3. The mean value of the normalized number of mobile agents required to restore completely the network's connectivity for (a) the plain and (b) the combined algorithm, after the termination of the network reconfiguration policy versus the network's size.

terms of efficient mobile agent placement compared to the plain algorithm. This is evident from the values of $\gamma_{w}$ recorded after the termination of the NetRec algorithm for all cases of network size and spatial topology examined. More precisely the normalized number of mobile agents required after the termination of the NetRec algorithm to reduce the number of clusters into 1 , is less when the combined algorithm is selected to calculate the number of cluster to be joined. Especially for the small and medium size networks $(7 \leq N \leq 29)$, the combined algorithm yields extremely satisfactory results related to $\gamma_{w}$, since its mean value remains below 0.67. Moreover, the maximum values of $\gamma_{w}$ recorded for the plain algorithm indicate an ineffective manner of handling the limited resources available to reduce the number of isolated clusters, since it remains above 0.8 , with the worst case $\left(\max \gamma_{w}=0.99\right)$ observed for a medium-sized network $(N=29)$. On the contrary, the combination of more that two clusters, under the constraint of a neighborhood determined by a distance weight factor framework, appears more efficient, since it manage to retain the maximum value of $\gamma_{w}$ below 0.87 .

The normalized blind region $\gamma_{B}$ is defined as the ratio of the network's blind region $\mathbf{A}\left(\mathbf{P}^{b}\right)$ and the total area within the bounds of the geographical span $\mathbf{G}_{w} \mathbf{G}_{h}$. The normalized blind area for the network cases examined before and after the reconfiguration process is presented at Figure 4. The strict definition of connectivity among
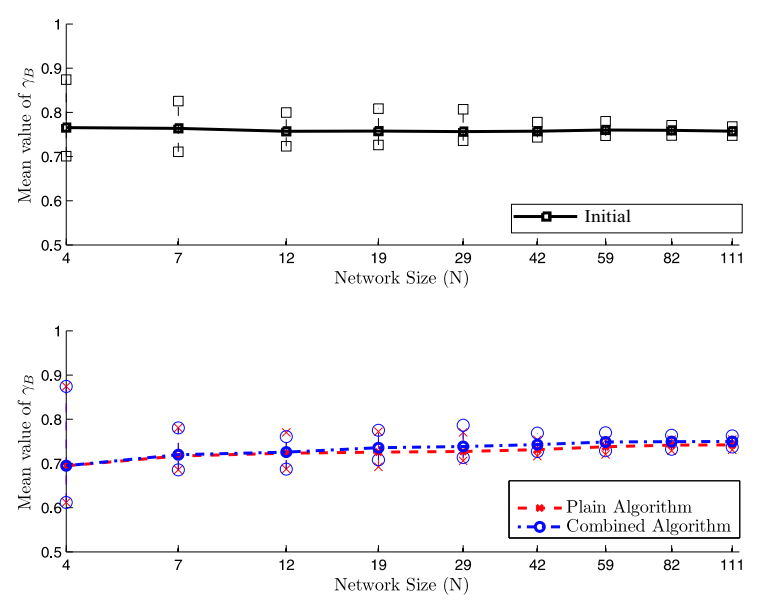

Fig. 4. The mean value of the normalized surface of blind region remaining before and after the termination of the mobile agent's placement process versus the network's size for (a) the plain and (b) the combined algorithm.

two nodes, described by (2), requires at least $50 \%$ overlapping of the transmission range areas. The impact of this basic requirement for connectivity is obvious when evaluating the improvement that the process of placing the mobile agents has to offer to the blind region coverage. More precisely, the mean value of $\gamma_{B}$ remains within 0.7 and 0.75 for all network cases examined, after the termination of the mobile agent placement process. These values recorded for $\gamma_{B}$ indicate no significant improvement on the blind region coverage, when compared to the status of the network before the introduction of mobile agents into the Voronoi-modeled network, where $0.75 \leq \gamma_{B} \leq 0.77$. Moreover, the variations of the mean value of $\gamma_{B}$ among the implementation of the plain and combined algorithm remain below 0.015 for all the network cases examined. These results are expected, since the cost functions implemented for the plain and combined algorithm do not involve the blind region coverage metric.

An example of the mobile agent deployment based on the plain and combined algorithm is highlighted at Figure 5. As shown in Figure 5(a), during the network's initial status, the number of isolated clusters is $M=17$, while the maximum cardinality observed is $L=2$. The positioning of the mobile agents based on the plain algorithm (Figure 5(b)) manages to reduce the number of clusters into $M=16$, while the maximum cardinality achieved is $L=3$. Finally, the implementation of the combined algorithm (Figure 5(c)) manages to connect 4 isolated clusters, resulting into $M=13$, while after the termination of the algorithm the cardinality of the most "popular" is $L=5$. 
(a)

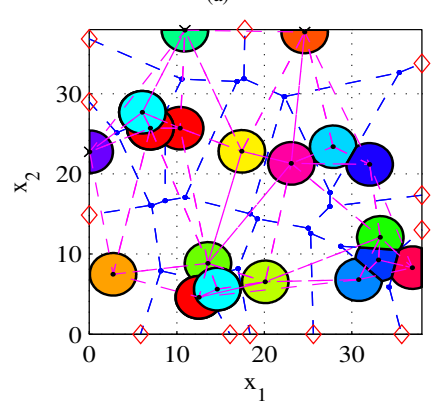

(b)

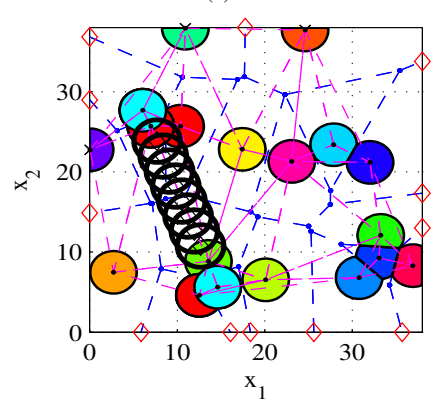

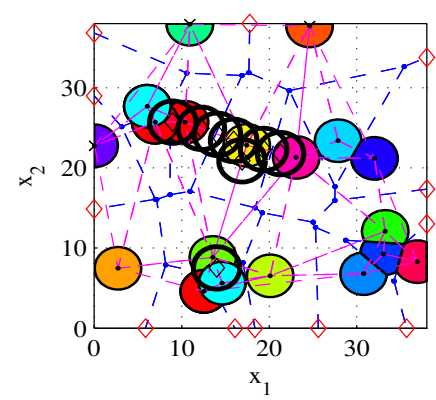

Fig. 5. A hWSN network reconfiguration example $(N=19)$, based on the plain (b) and combined (c) algorithm. The mobile agents are represented with the diamond $(\diamond)$ mark.

\section{CONCLUSiOnS}

In this work improvements on the original reconfiguration concept for WSN presented in [19] were presented. Under the assumption of static nodes with known coordinates, the spatial attributes were derived based on a Voronoi diagram. The primary focus of the network is the establishment of the connectivity among the operational nodes. Towards this direction, two algorithms for the introduction of mobile agents are evaluated. The simulation scenarios, which were developed within bounded geographical areas, varied in terms of network size and nodal spatial distribution, while the number of mobile agents available was limited and depended on the network's size examined.

The simulation results investigated the efficiency of the proposed algorithms in terms of several performance metrics and highlighted the advantages of the combined algorithm over the plain algorithm. The limitation on the number of mobile agents available, combined with the requirement for bidirectional communication link, resulted in slight improvement on the coverage of the network's blind regions for both algorithms compared to the network's initial status. However, the implementation of the combined algorithm has offered better results in terms of connectivity status after the termination of the reconfiguration process, and efficient "consumption" of mobile agents, than the plain algorithm.

\section{REFERENCES}

[1] I. F. Akyildiz, X. Wang, and W. Wang, "Wireless mesh networks: a survey," Computer Networks, vol. 47, pp. 445-487, 2005.

[2] F. Zhao and L. Guibas, Wireless Sensor Networks. Elsevier, 2004.

[3] S. S. Iyengar and R. R. Brooks, Distributed Sensor Networks, ser. CRC Computer and Information Science. Chapman and Hall, 2005.

[4] J. Scholtz, J. Young, J. L. Drury, and H. A. Yanco, "Evaluation of human-robot interaction awareness in search and rescue," in Proceedings of the International Conference on Robotics and Automation (ICRA), New Orleans, LA, USA, 2004.

[5] I. Vasilescu, K. Kotay, D. Rus, M. Dunbabin, and P. Corke, "Data collection, storage and retrieval with an underwater sensor network," in Proceedings of ACM Sensys, San Diego, 2005.
[6] M. Dunbabin, P. Corke, I. Vasilescu, and D. Rus, "Data muling over underwater wireless sensor networks using an autonomous underwater vehicle," in Proceedings of the IEEE International Conference on Robotics and Automation, Orlando, FL, USA, May 2006, pp. 2091-2098.

[7] Q. Fang, J. Liuy, L. Guibas, and F. Zhaox, "RoamHBA: Maintaining group connectivity in sensor networks," in Proceedings of the IPSN, Berkeley, CA, USA, April 2004.

[8] S. A. Aldosari and J. M. F. Moura, "Distributed detection in sensor networks: Connectivity Graph and Small World Networks," in Proceending of The Asilomar Conference on Signals, Systems, and Computers, Pacific Groove, CA, USA, October 2005, pp. 230-234.

[9] X. Wang, G. Xing, Y. Zhang, C. Lu, R. Pless, and C. Gill, "Integrated coverage and connectivity configuration in wireless sensor networks," in Proceedings of ACM SenSys, Los Angeles, CA, USA, November 2003.

[10] V. D. Silva and R. Ghrist, "Coverage in persistent networks via persistent homology," Algebraic and Geometric Topology, vol. 7, p. 339358, 2007.

[11] F. Preparata and M. Shamos, Computational Geometry: An Introduction. Springer-Verlag, 1985.

[12] S. Megerian, F. Koushanfar, M. Potkonjak, and M. B. Srivastava, "Worst and best-case coverage in sensor networks," IEEE Trancation on Mobile Computing, vol. 4, no. 1, pp. 84-92, January 2005

[13] G. Wang, G. Cao, and T. F. L. Porta, "Movement-assisted network deployment," IEEE Transactions on Mobile Computing, vol. 5, no. 6, pp. 640-652, June 2006.

[14] A. M.-C. So and Y. Ye, "On solving coverage problems in a wireless sensor network using voronoi diagrams," in Proceedings of the 1st Workshop on Internet and Network Economics, ser. LNCS 3828, 2005, pp. 584-593.

[15] X. Y. Li, P. J. Wan, and O. Frieder, "Coverage in wireless ad-hoc sensor networks," IEEE Transactions on Computers, vol. 52, no. 6, pp. 753-763, June 2003.

[16] A. Muhammad and A. Jadbabaie, "Decentralized computation of homology groups in networks by gossip," in Proceedings of American Control Conference, New York City, NY, USA, July 2007, pp. 3438-3443.

[17] _ Dynamic coverage verification in Mobile Sensor Networks Via Switched Higher Order Laplacians, O. Broch, Ed. MIT Press, 2007.

[18] S. Martínez, J. Cortés, and F. Bullo, "Motion coordination with distributed information," Control Systems Magazine, vol. 27, no. 4 , pp. $75-88$, August 2007.

[19] A. Panousopoulou and A. Tzes, "Utilization of mobile agents for voronoi-based heterogeneous wireless sensor network reconfiguration," in Proceedings of European Control Conference, Kos Island, Greece, July 2007, pp. 4276-4284.

[20] T. S. Rappaport, Wireless Communications: Principles and Practice. Prentice Hall, 1996. 